\title{
A report on a project to introduce computation into a Modern Physics course and laboratory
}

\section{Dr. Marie Lopez del Puerto, University of St. Thomas}

Marie Lopez del Puerto completed her B.S. in physics at Universidad de las Americas, Puebla, in Puebla, Mexico, and her Ph.D. in physics at the University of Minnesota - Twin Cities, in Minneapolis, MN. She is currently an Associate Professor in the Physics Department at the University of St. Thomas in St. Paul, MN. Her research interests include the structural, optical and electronic properties of nanoscale systems, computational physics, and physics and engineering education. 


\section{A report on a project to introduce computation into a Modern Physics course and laboratory}

The importance of computational physics both as a discipline and as a component of the undergraduate curriculum has been recognized for some time. The challenge lies in creating course materials that introduce students to computational physics with problems that are meaningful and challenging, yet are neither overwhelming to the students nor take substantial time from the more traditional theoretical and experimental components of the course(s). In a previous paper we proposed an approach to introducing computational physics in the undergraduate curriculum by

blending computation and experimentation in the Modern Physics course and laboratory with materials that discuss contemporary physics subjects (quantum dots, LASERs, superconductivity, etc). In this paper we report on the homework problems and laboratories that have been developed as part of this project and the successes and challenges in implementing these materials, and we point to future work to be done in this area.

In 2011, the American Association of Physics Teachers approved a "Statement on Computational Physics"" that says: "The American Association of Physics Teachers urges that every physics and astronomy department provide its majors and potential majors with appropriate instruction in computational physics." With the rationale that "computational physics has become a third way of doing physics and complements traditional modes of theoretical and experimental physics." Similarly, the ABET program criteria for Electrical and Computer Engineering states: "The curriculum must include . . . engineering topics (including computing science) necessary to analyze and design complex electrical and electronic devices, software, and systems containing hardware and software components. ${ }^{2,}$

The approach we have taken in the Physics Department at the University of St. Thomas to address the issues raised in the above statements is to integrate computation throughout the curriculum. The rationale behind this approach is that students need time to develop and practice their computational skills. Students become comfortable using computation through continued practice. By embedding computation in different courses students see the wide applicability of computational techniques and come to see it as one more tool in their arsenal - on par with theoretical and experimental skills.

Students are first exposed to computation in the physics curriculum in the sophomore-level Applications of Modern Physics course and laboratory. This course is required for both Physics and Electrical Engineering majors; it is also often taken by other science majors as part of the Physics minor. The course has been redeveloped over the past six years with the following goals:

1. To ease the transition between lower-level and upper-level physics courses as the course material becomes more abstract and the mathematics more sophisticated.

2. To modernize the course by discussing the physics behind contemporary applications such as LASERs, quantum dots and other nano-scale systems, diodes, and superconductors. 
3. To introduce students to computational physics and the interplay between theory, computation and experiment. Validation experiments engage students with the material as they test the validity of the theoretical and computational models they develop.

At the University of St. Thomas the introductory (100-level) physics sequence is taught using a modified workshop format with three 2.5-hour class sessions per week. Lecture, discussion, problem solving, and lab work are woven into the period, with students often working in groups of three to four. All of our 200-level and above courses, on the other hand, have separate lecture and lab periods, with limited time for guided problem solving in class. Thus, students often struggled with the transition from lower-level to upper-level courses. In addition, students in the Applications of Modern Physics course would often comment on the disconnect between lecture and lab, which were taught by different instructors before 2010. To address this issues the same instructor started teaching both the lecture and laboratory sections in Spring 2010 and some laboratory time was allocated to guided inquiry using PhET simulations ${ }^{3}$ and materials modified from those developed at the University of Colorado at Boulder ${ }^{4}$.

To address goals 2 and 3 , the redeveloped course and laboratory have a materials science focus. The properties of single atoms and collections of atoms are studied with an eye toward understanding real-world applications. Labs closely tie in to what is being discussed in lecture and introduce students to computational modeling using MATLAB. We chose this particular highlevel language for two reasons: (1) both Physics and Electrical Engineering students use it throughout their upper-level courses and (2) it is widely used in industry and academic research. Brief, illustrative experiments are used to test the validity of the models and to highlight the connection between theory, computation, and experiment that is pervasive in contemporary research. A sample laboratory schedule is shown below:

\begin{tabular}{|c|l|}
\hline Week & \\
\hline 1 & Lab 1: Intro to MATLAB \\
\hline 2 & Lab 2: Classical Statistics Simulation \\
\hline 3 & Lab 3: Classical Statistics Experiment \\
\hline 4 & EXAM 1 \\
\hline 5 & Lab 4: Photoelectric Effect \\
\hline 6 & Lab 5: Electromagnetic Waves \\
\hline 7 & Lab 6: Quantum Mechanics: Tunneling \\
\hline 8 & EXAM 2 \\
\hline 9 & Lab 7: Discharge Lamps \\
\hline 10 & Lab 8: LASERs \\
\hline 11 & EXAM 3 \\
\hline 12 & Lab 9: Quantum Dots Experiment \\
\hline 13 & Lab 10: Quantum Dots: Particle-in-a-Box Model \\
\hline 14 & Lab 11: Thermocouples and Superconductors \\
\hline
\end{tabular}

A full description of the laboratories is given in references 5 and 6 . 
Students come into this course with a wide range of abilities in computational programming, though a majority of students do have some experience. In 2014, $86 \%$ of students reported having already taken a college-level computer science course and $79 \%$ reported having previous experience with MATLAB. Only $7 \%$ of students reported having no previous programming experience. To account for this range of experiences the first three laboratories, which focus on classical statistics and two-step systems, serve as an introduction to MATLAB and the use of simulations in physics.

We have found that even students who are familiar with programming struggle to translate physics equations into a working algorithm. Students need repeated practice on debugging and testing code with 'experts' (the instructor and teaching assistant(s)) available to help, particularly in the beginning of the semester. Having enough support the first few weeks is key to a successful experience as students get easily frustrated when their program does not run and they do not understand the error messages. Currently laboratories of 12 to 16 students are staffed by the instructor plus one or two teaching assistants. Students work independently, but they sit in tables of four and are encouraged to discuss the lab with each other.

In order to have students practice the computational skills they are acquiring and to further tie the lecture and laboratory together, we have developed short homework exercises that closely tie to what students are learning in lecture and in lab. For example, after students fill out an online class survey, the following homework problem is assigned (part of the classical statistics unit):

\section{PHYS 225 - Homework \#2}

Problem \#4

The following is a list of distances your classmates live from campus, where 0 represents that they live on campus. Distances are in miles.

$d=[21.9,0,10,0,16,3.4,1.8,0,0.7,0.3,0,0,15,10,0,0,0$, $0,13,0.12,9,32.6,0,4,0.5,4,0,4,0,1.7,8,0.4,25,1,47$, $2.8]$

a) Use the hist() command to generate a histogram of d.

b) How does the histogram you just generated compare to your prediction from Homework \#1? Explain any discrepancies.

c) What is the mean distance students in PHYS 225 live from campus?

d) What is the median distance students in PHYS 225 live from campus?

e) If you were to randomly ask a student in class where he/she lives, what is the probability he/she lives on campus?

f) What is the probability he/she lives more than 20 miles from campus?

Publish the file, check for correct formatting (does the text show up as text? does the plot have axes labels?), print the file, and turn it in with the rest of this homework packet. 
We want students to recognize the importance of the three ways of doing physics (theoretical, experimental, and computational), in order to do so each exam in this course includes at least two of the three components. To facilitate this, exams are taken during a laboratory period and consist of two parts. Part one is a traditional exam with both conceptual questions and problems. Part two is a practical exam with a problem that involves either computation, or both experiment and computation. This second part is based on a lab that students have previously completed so they do not have to start programming from scratch, and they use the same or very similar experimental techniques.

The following is an example of Part II of the exam that corresponds to the unit that includes properties of single atoms. Before the exam students have completed two labs in this unit: discharge lamps and LASERs.

\section{PHYS 225 - Exam \#3 Part II}

- Use a spectrometer and SpectraSuite to record the spectra of a mercury discharge lamp. Save the data as a tab delimited, no header, text file.

- Use a spectrometer and SpectraSuite to record the spectra of the overhead fluorescent lights. Save the data as a tab delimited, no header, text file.

Q1. Using MATLAB, read in your experimental data and plot it. There should be two plots in the same figure window, one above the other. The plots should have the same $x$-axes range and should all have intensities normalized to 1 so that you can easily compare them.

Q2. Use MATLAB to find the wavelengths that correspond to the 7 highest intensity peaks in your experimental data for the mercury discharge lamp.

Q3. Use MATLAB to find the wavelengths that correspond to the 7 highest intensity peaks in your experimental data for the fluorescent lights.

Q4. In MATLAB, make a data table with two columns, one for the wavelengths calculated in Q3, and the other for those in Q4. Columns should be sorted in order of increasing wavelength. Wavelengths that are similar (i.e. within $\sim 1 \mathrm{~nm}$ ) across columns should be on the same row, even if you have to leave some spaces empty.

Q5. Using the concepts we have learned in class along with the data you took today, describe how you would convince one of your friends (who has not taken PHYS 225, but has taken introductory physics) that there is or that there is no mercury in the overhead fluorescent lights.

- Publish your script, print it and staple it to this handout. 
Changes to the lecture portion of the course started in Spring 2009. A new book was selected (S.O. Kasap, Principles of Electronic Materials and Devices, McGraw-Hill, $3^{\text {rd }}$ ed.) and in-class multiple-choice questions were introduced in order to promote discussion and give the instructor feedback on the students' understanding of the material. Changes to the laboratory portion started in Spring 2010, when the same instructor was assigned to teach both the course and the two associated laboratory sections. It is known ${ }^{7}$ that it takes time for changes to the curriculum to be accepted, and that was the case with this course. In the beginning students were reluctant to see computation as part of a physics lab. However, after the third iteration of the course, there was a noticeable change in attitudes: students began to request more computation in the labs that did not have as much of it.

Lessons learned:

- Including computational and experimental sections in exams encourages students to complete the laboratories thoroughly and to make sure they have a good understanding of the material and techniques used.

- Computational laboratories are easy to adjust for different skill levels. Advanced students enjoy extra challenges.

- Computational homework highlights the usefulness of the skills the students are gaining, gives them more practice, and helps to tie the lecture and lab together.

- Having sufficient support during the first few weeks of laboratory is essential in preventing student frustration with coding and debugging. The past three years there has been one instructor and one teaching assistant in each lab section. This semester (Spring 2015) we are experimenting with having two teaching assistants in each lab section during the first three weeks of the semester.

\section{Challenges:}

- As enrollment has grown (shown in Fig. 1), one instructor is no longer able to teach both lecture and all lab sections. In Spring 2015 there will be two lecture sections and three lab sections taught by two instructors. Thus, it will be more difficult to maintain the close coordination between lecture and lab.

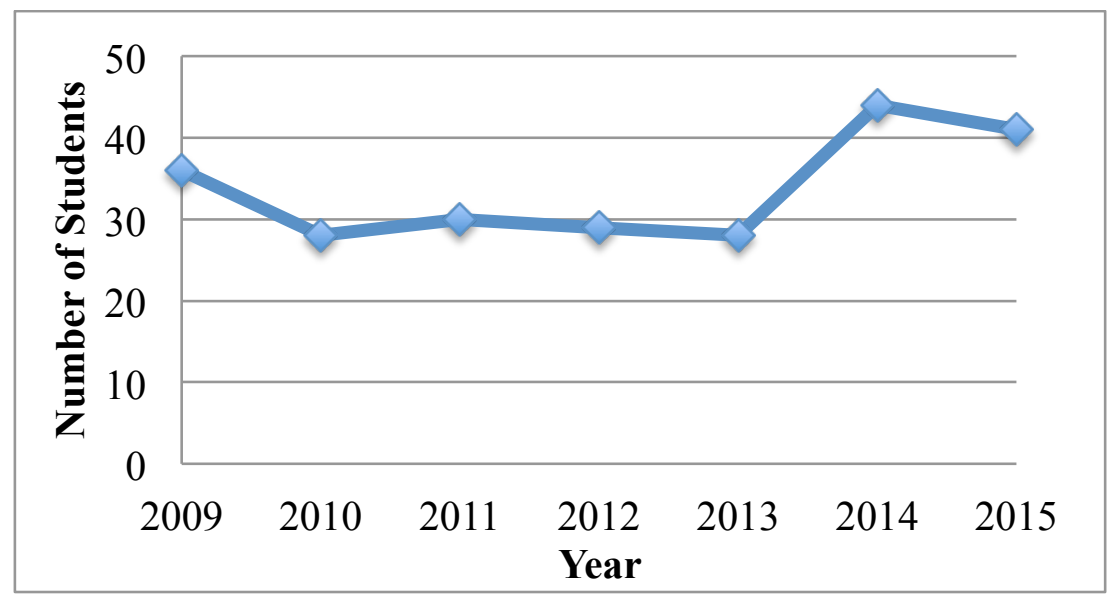

Fig. 1: Enrollment in Applications of Modern Physics, 2009-2015 
- $\quad$ Finding teaching assistants for 3.5 hour laboratory sections can be difficult. Teaching assistants are typically upper-level science and engineering students who have taken the course before, and scheduling conflicts with their classes, laboratories, and internships are common.

Future work:

- $\quad$ Standardized tests $\left(\mathrm{QMCS}^{8}\right.$ and $\left.\mathrm{CLASS}^{9}\right)$ as well as questionnaires have been given to students each year. Analysis on these data is ongoing.

- More computational homework problems need to be developed. The challenge is in finding problems that are relevant, use the skills the students are working on, and are doable in a similar amount of time as a regular homework problem.

- The course materials and laboratories are continually being updated to keep them current.

The laboratories developed as part of this project have been shared with faculty at other institutions and are available by contacting the author.

\section{Bibliography}

[1] AAPT, Statement on Computational Physics http://www.aapt.org/Resources/policy/Statement-onComputational-Physics.cfm

[2] ABET, Criteria for Accrediting Engineering Programs, 2012-2013 http://www.abet.org/DisplayTemplates/DocsHandbook.aspx?id=3143

[3] The Physics Education Technology project (PhET) interactive simulations, http://phet.colorado.edu

[4] S. B. McKagan, K. K. Perkins and C. E. Wieman, "Reforming a large lecture modern physics course for engineering majors using a PER-based design,” PERC Proceedings 2006, AIP Press. Melville, NY (2006).

[5] M. Lopez del Puerto, Applications of Modern Physics: A Sophomore-Level Course and Laboratory for Electrical Engineering Students, Proceedings of the ASEE Annual Conference, San Antonio, Texas, American Society for Engineering Education (2012).

[6] Curriculum Development for PHYS 225: Applications of Modern Physics, http://ida.phys.stthomas.edu/PHYS225curriculum

[7] C. Henderson, A. Beach and M. Famiano, "Promoting instructional change via co-teaching," American Journal of Physics, 77, 274 (2009).

[8] The Quantum Mechanics Conceptual Survey (QMCS) was developed by Sarah McKagan, Carl Wieman, and Kathy Perkins at the University of Colorado at Boulder.

[9] The Colorado Learning about Science Survey (CLASS) was developed by Wendy Adams, Noah Finkelstein, Kathy Perkins, Noah Podolefsky and Carl Wieman at the University of Colorado at Boulder. 